\title{
Desmidiées en tant qu'indicateurs de la qualité des eaux dans la région de Marrakech (Maroc)
}

\author{
K. Mouhri1 \\ J. Darleyl \\ R. Le Cohu ${ }^{2}$ \\ J. Loudiki ${ }^{1}$
}

Mots clés : Algues, Desmidiées, Maroc, Dayas, bassins permanents, réservoir, propriétés chimiques.

Trente-sept sites de la région de Marrakech (dayas, bassins permanents et réservoirs) ont été prospectés régulièrement pendant un an. Les analyses factorielles de correspondance, qu'elles soient réalisées à partir des descripteurs ou à partir des facteurs biotiques (133 taxons de Desmidiées), permettent de visualiser les mêmes typologies au sein des biotopes étudiés. A la lumière de ces résultats, les Desmidiées apparaissent comme de bons indicateurs dans l'appréciation de la qualité chimique des eaux du Sud du Maroc.

Desmids as indicators of water quality in the Marrakech region (Morocco).

Keywords : Algae, Desmids, Dayas, permanent basin, reservoir, chemical characters.

Thirty seven diverse sites of the Marrakech Region (Morocco) were investigated fornightly or monthly over one year. Two factorial corr espondence analyses demonstrated the similar typologies; one of them was based only on the environmental factors, the second one on the biotic parameters (133 Desmid taxons). It is concluded from these results that, the Desmids seem to be good biological indicators for evaluating the chemical water quality in South Morocco.

\section{Introduction}

Les recherches sur les algues réalisées à ce jour au Maroc sont variées : inventaires (Belloc 1893 \& 1896, Harriot 1913, Mercier 1922, Gauthier-Lièvre 1931 \& 1951, Gatteffosse \& Werner 1935, Werner 1934, Bourrelly \& Gayral 1951a \& 1951b, Somers $1972 \ldots$..) ; études écologiques (Gayral 1954, Sasson 1959) ; descriptions d'espèces nouvelles pour le Maroc ou pour la science (Bourrelly \& Gayral 1950, Darley \& Couté 1983) ; études de la biomasse et de la production primaire (Derraz 1987, Marqué \& Dauta 1987).

La présence de desmidiées (c'est-à-dire Mesotaeniacées et Desmidiacées) a souvent été signalée, mais aucune étude exhaustive de ce grand ensemble n'a

1. Faculté des Sciences, Département de Biologie, BP. s 15 , Marrakech, Maroc.

2. Laboratoire d'Hydrobiologie, UA 695 du CNRS, Université Paul Sabatier, 118, route de Narbonne, 31062 Toulouse Cèdex, France. été entreprise ; pourtant l'écologie des desmidiées des régions tropicales est différente de celle des pays tempérés en ce sens que plus on se rapproche de l'équateur et plus ces algues semblent s'adapter ou même se complaire en milieu alcalin (Van Oye 1949, Talling \& Talling 1965). Les desmidiées sont en outre des indicateurs délicats de changement de l'environnement (Lund 1971, Bourrelly 1972).

Ces constatations nous ont amené, du fait de la situation géographique du Maroc entre tropique et zones tempérées, à entreprendre l'étude des populations desmidiales planctoniques et périphytiques, sur le plan qualitatif et quantitatif, pour connaître leur répartition dans différents écosystèmes aquatiques continentaux du Maroc central.

\section{Matériel et méthodes}

37 sites répartis dans la région de Marrakech (fig. 1) ont été prospectés pendant le cycle annuel 1985-1986 (Mouhri 1987). Les prélèvements d'eau destinés aux analyses physico-chimiques et les 


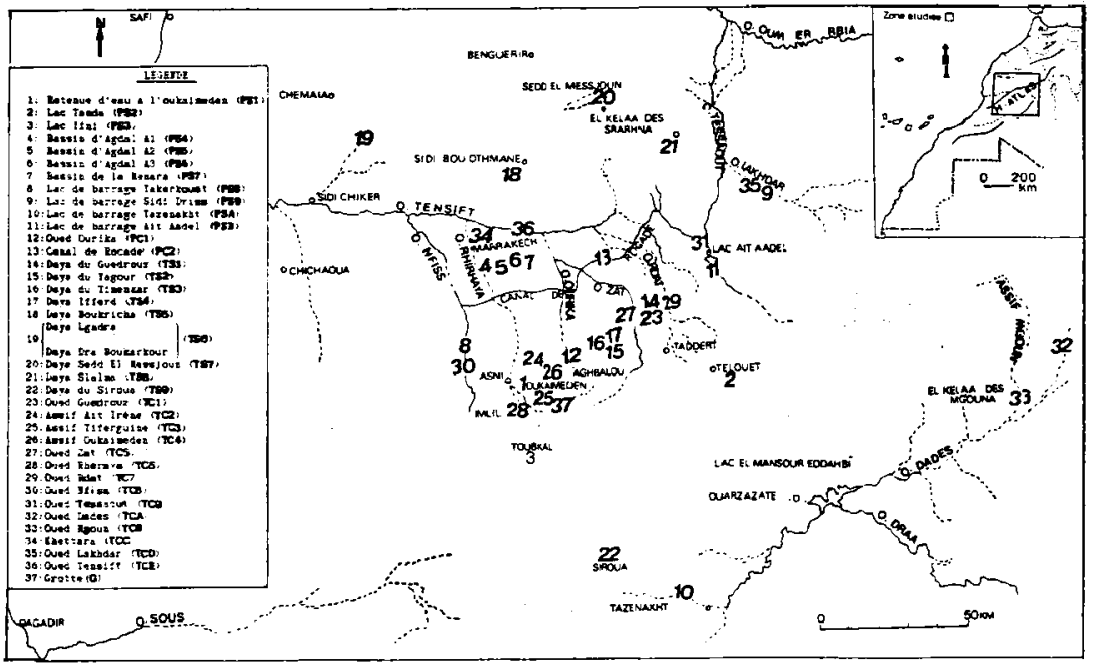

Fig. I : Situation géographique des sites étudiès.

récoltes d'algues (filet à plancton, grattage des supports existants, expression de végétaux aquatiques) ont été effectués tous les 15 jours (mares temporaires ou dayas) et tous les mois (milieux permanents).

L'ensemble des échantilions d'algues périphytiques et planctoniques ont fait l'objet d'une analyse qualitative et semi-quantitative ( $\%$ d'abondance). Pour cette dernière, un sous-échantillonnage a permis d'évaluer le nombre minimum d'individus à compter. Cette analyse a été réalisée sur trois types de milieux :

- une mare temporaire de haute montagne : Guedrouz, choisie pour sa grande richesse spécifique (fig. 2a). 2b).

- Un bassin permanent de plaine: Agdal (fig.

- Un lac de barrage de montagne : Oukaimeden (fig. 2c).

Les courbes obtenues (fig. 2) montrent que la stabilisation de la moyenne et des écarts type est atteinte à partir de 40 individus comptés (IC) dans le cas d'un peuplement peu diversifié. Par contre, pour les peuplements très diversifiés, riches en espèces de desmidiées, elle n'est acquise qu'à partir du comptage de 200 individus.

En outre, une analyse quantitative (Utermohl 1958) a été réalisée sur les desmidiées planctoniques de cinq stations différentes afin de connaître la richesse en espèces et en individus de leur peuplement desmidial :

- daya de montagne : Guedrouz (TS1)

- lac de barrage : Oukaimeden (PS1)

- bassins de Marrakech : Agdal et Ménara (PS4 et PS7)

- daya des jbilets : Boukricha (TS5).

Dans le but de connaître la répartition des espèces de desmidiées dans ces différents hydrosystèmes et de mettre en évidence leur rôle d'indicateurs biologiques de la qualité hydrochimique, des analyses multivariées (AFC) (Cordier 1969, Benzecri \& Lebart 1970) ont été effectuées sur les données abiotiques et biotiques. 

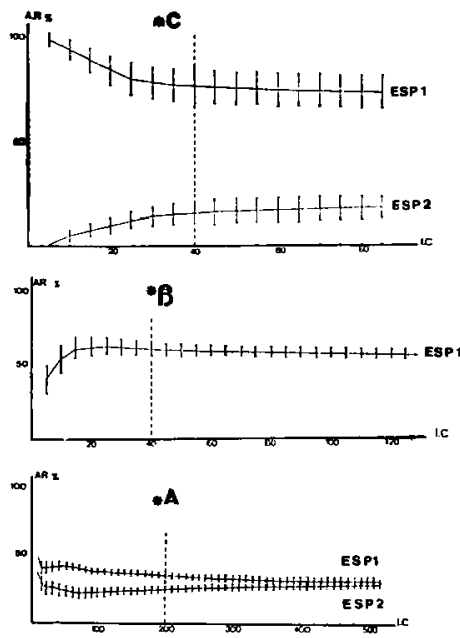

Fig. 2 : Détermination du nombre minimum d'individus comptés dans l'analyse semi-quantitative de 3 milieux différents ; $\mathbf{A R}=$ Abondance relative $; \mathrm{IC}=$ Individus comptés $; 2-\mathbf{a}$ : daya Guedrouz (TS1), 2-b : bassin d'Agda] (PS4), 2-c : lac Oukaimeden (PSI).

\section{Résultats et discussion}

\subsection{Inventaire des desmidiées}

133 taxons, dont 85 nouveaux pour le Maroc, ont été inventoriés dans les 37 écosystèmes aquatiques étudiés. Leur liste est donnée pages 112 et 113 .

Les sigles GMO, GBL, GBR, etc... correspondent au codage des espèces utilisé dans l'AFC.

Chaque espèce nouvelle pour le Maroc est signalée par **.

\subsection{Analyse quantitative}

La figure 3 montre que la daya de Gtiedrouz constitue le biotope le plus riche en desmidiées, soit 162.000 cellules/litre. Le peuplement y est dominé par deux espèces de Gonatozygon et une espèce de Cosmarium :

G. monotaenium : 46.675 cellules/litre $(\neq 29 \%$

G. brebissonii : 26.310 celtules/litre $(\neq 16 \%)$

et C. subcapitulum : 34.388 cellules/litre $(\neq 21 \%)$.

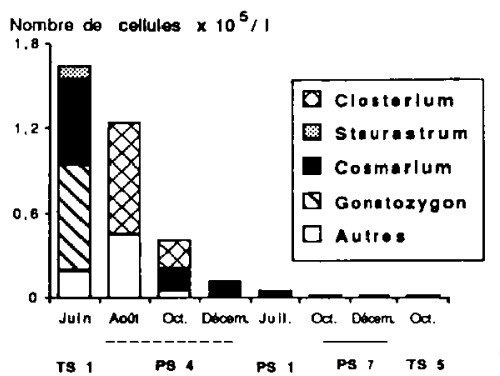

Fig. 3 : Résultats de l'analyse quantitative des Desmidiees au cours de l'année 1986 dans 5 écosystèmes différents : daya Guedrouz (TS1), daya des Jbilets (TS5), bassins d'Agdal (PS4) et de la Ménéra (PS3) et le lac Oukaimeden (PSI). Les résultats sont exprimés en nombre de cellules par litre.

Le peuplement algal du bassin d'Agdal est dominé quant à lui par une seule espèce : Closterium dianae ; cette espèce représente jusqu'à $70 \%$ du peuplement algal (83 $200 \mathrm{cell} . / \mathrm{ml}$ ) au mois d'août. Les autres milieux sont pauvres en desmidiées avec une dominance du genre Cosmarium.

\subsection{Typologie des milieux étudiés}

\subsubsection{REPARTITION DES BIOTOPES EN FONCTION DES CRITERES MESOLOGIQUES (AFC)}

Une analyse factorielle des correspondances a été effectuée sur une matrice des données comportant 21 paramètres abiotiques de 23 stations : température de l'air et de l'eau, pH, conductivité, turbidité, oxygène dissous, profondeur de l'eau, matières en suspension, nitrites, nitrates, ions ammonium, orthophosphates, alcalinité, dureté totale, calcium, magnésium, chlorures, durée de mise en eau, étage bioclimatique, variante d'hiver (froid, frais ou chaud), altitude. La classifjcation hiérarchique $(\mathrm{CH})$ permet de subdiviser les milieux en 4 groupes (G1, G2, G3, G4) et selon 3 gradients (fig. 4) : 


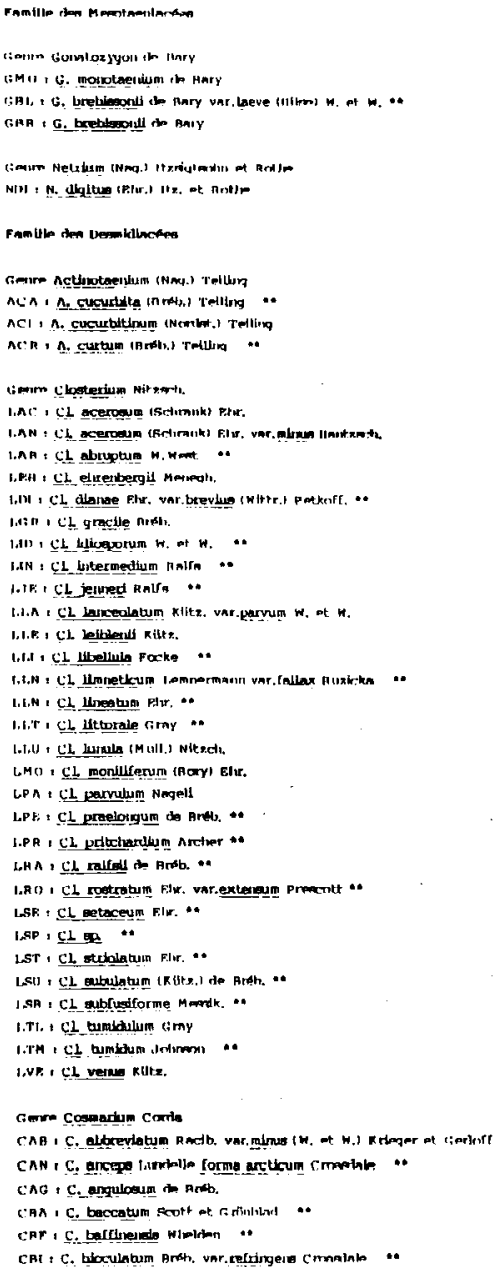

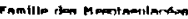

cimin Gonalox zyoll to linry

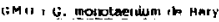

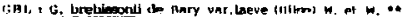

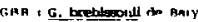

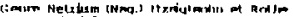

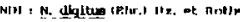

Fantiln den Vermklincter

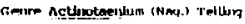

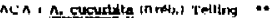

nCI i n. cucurbitimum inembot,t Teiling

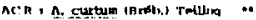

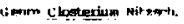

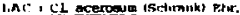

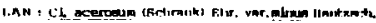

1.An : CL Albaptupr $H, H$ mot. $\cdots$

i,kn + CL elirenbergh Marnalh.

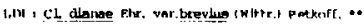

I,A3 I CLl gractle niflr.

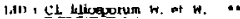

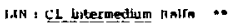

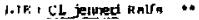

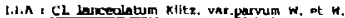

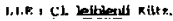

I.T.I , cl L Ltelluin Focke -.

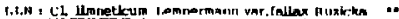

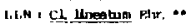

l, f, I L Iftoraie Gray in

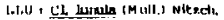

LMO i CL monjlifenm thorl Elur.

CPA t CL parvulum Negell

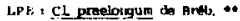

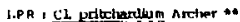

LHA, CL ralfell at Arib. "A

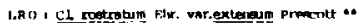

ISR : CL Eetoceum FIr. *

ISP , CLEA *

LST, Cl, studolatutn Flor. $\cdots$

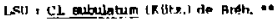

I SA , Cl abrugiforme Mardk. " "

I.T1, + CL mombulum timy

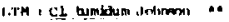

l, Un, L 1 veras Rutz.

Gmor Cosanarun Coris

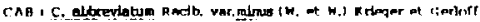

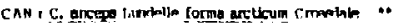

cac, C. expulonem in andb.

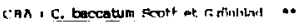

car, C. barrinends wimirbon *

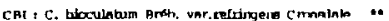

Cuk 1 c. tineme hardit. $\cdots$

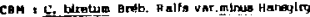

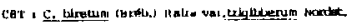

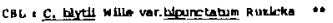

CBO : C, bookdl willw

$\mathrm{CBH}, \mathrm{C}$, botritis meregh

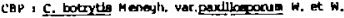

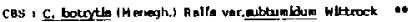

cCe 1 c. calcareura witrok

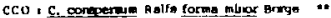

CCN , c. constetenn bejponte -.

CFA, c. Eastdonim W. At $w, \cdots$

CFO, $\mathrm{C}$. fonthenum Nomst, ."

CPR , C, formoajum Hof. var, farthoretl Bolbt

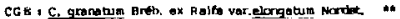

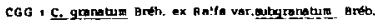

(พY) , $\mathrm{c}$, mande

CHO \& c. holmbers: lindell *o

CHA : $\mathrm{G}$, homavenge Gutwinak1 *

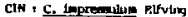

Cl, \& C. lowe (Groy) Narlat.

CLM I c. Latubore Lind, ? *

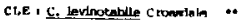

CMA I C. mote stron **

CHI) : C. mangaritatum (t,ind,) Roy et pleset

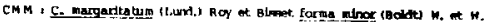

CMR : C. marocenup varticy et Cout

CHE + C. metieghinili end.

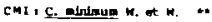

CNO 1 c, notablie B ont.

CPA : C. pectrodermun l,and. *.

CPH , C. ptaseolus Rolrt *

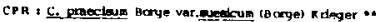

CPU * C. puedlum daneb.1 Arth, *

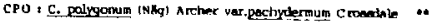

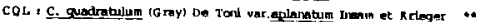

CQM : C. quadrotum Ralfi "*

CHR : C. Eggnellil wille

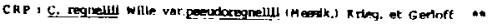

CRE : C. regred Relineh O

CRN : C. repiforme I Ralfin Anch.

CRT 1 C: rehoum IPertyl Rebh.

$\csc +$ C. expulorum acrye

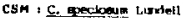

C8. : C. Eolnulifenum $W$. et W. *

C9O : c. mortelle var oubrudum $W$. et $W$. "*

CSR + C. Dubcrenatum Ilantsich **

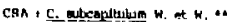

CSU : C., aboototum Nomint. $\cdots$

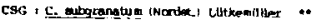

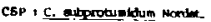

CBR I C. alkeinachll M modh. **

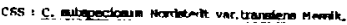

CTI t c. tinctue Ralf ".

cru : c, brolnll natb. * 


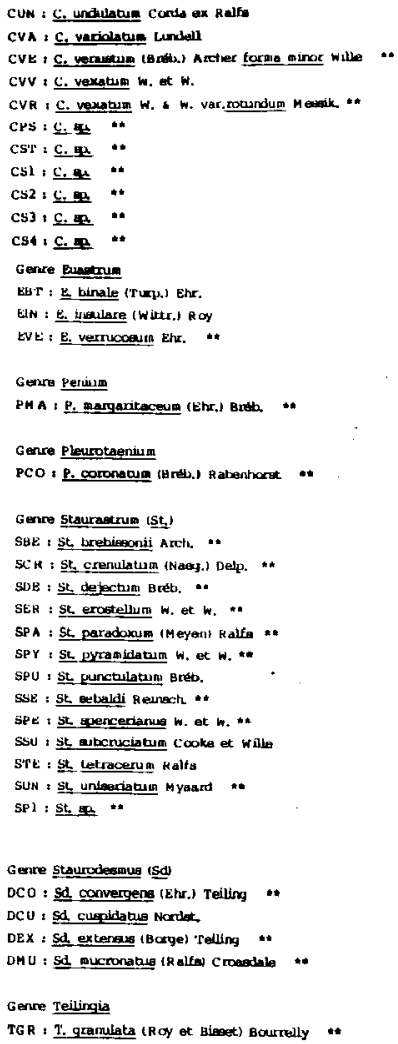

- un premier gradient (A) altitudinal et climatique oppose le groupe $\mathrm{G} 4$ aride de plaine au groupe Gl de haute altitude à climat froid ;

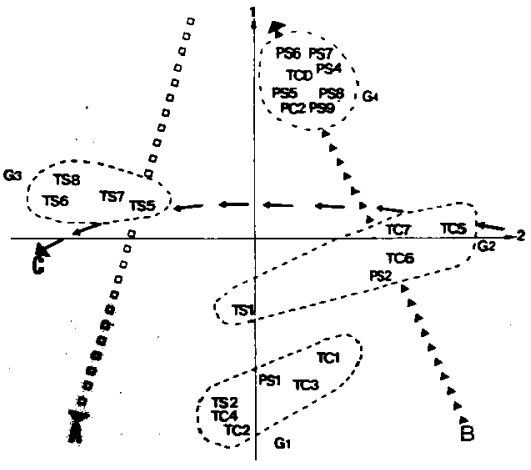

Fig. 4 : Analyse spatiale (AFC): Typologie des milieux étudiés dans l'espace des paramèt res abiotiques. A : gradient altitudinal et climatique. B : gradient de minéralisation de l'eau. $C$ : gradient de turbidité et de charge des eaux en matières en suspension.

- un second gradient (B) de minéralisation de l'eau oppose le premier groupe G1 de faible minéralisation, lac Oukaimeden (PS1), Yagour (TS2), Guedrouz (TC1), Assif Ait Irene (TC2), au groupe G4 caractérisé par des eaux assez dures, alcalines et assez fortement minéralisées, bassins de Marrakech (PS8), Sidi Driss (PS9), et Canal de Rocade (PC2) ;

- un troisième gradient (C) traduit la charge des eaux en matières en suspension associée au degré de turbidité : il oppose le groupe G2 caractérisé par des eaux faiblement turbides (Oueds du Haut-Atlas: TC5, TC6, TC7) au groupe G3 formé par les eaux peu profondes des mares temporaires des Jbilets (TS5, TS6, TS7) et de la Bahira (TS8) qui ne sont jamais transparentes (piétinement fréquent du bétail, raclage permanent du sol par les Triops...). 


\subsubsection{REPARTITION DES BIOTOPES EN FONCTION DES FACTEURS BIOTIQUES}

L'étude de la distribution des espèces dans le plan factoriel (1-2) met en évidence l'existence de 5 noyaux d'espèces et 5 noyaux de stations (fig. 5 et 6) :

- Le noyau 1 est séparé en deux sous-unités ; la première (N1-1) renferme des espèces affines au système daya, récoltées dans les mares temporaires de montagne (Guedrouz, Yagour : Groupe G1) dont la position sur l'axe 1 , nettement positive, les lie à des conditions de milieu bien définies, à savoir : faible minéralisation, $\mathrm{pH}$ acide ou voisin de la neutralité et phase d'assec obligatoire ; ce sont Cosmarium humile, C. subcapitulum, Actinotaenium curtum, Teilingia granulata, Gonatozygon brebissonii.

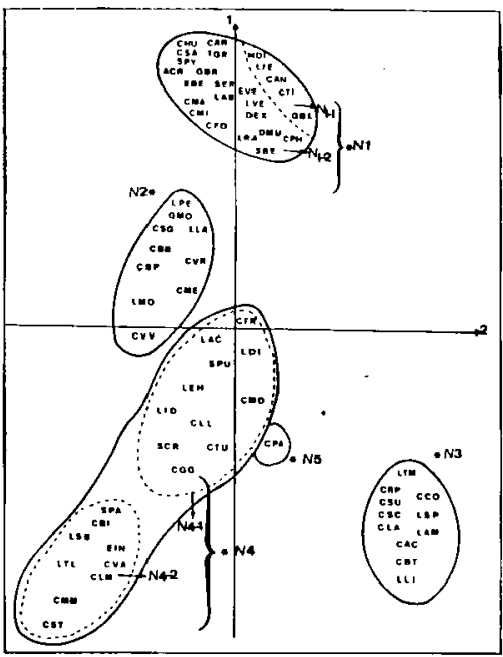

Fig. 5 : Analyse spatiale (AFC) : Projection des différents taxons dans le plan factoriel (1-2). $\mathrm{N}=$ groupe d'especes.

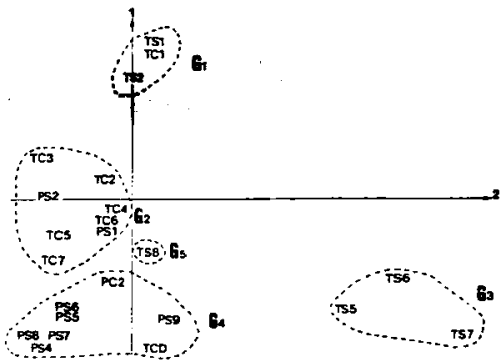

Fig. 6 : Analyse spatiale (AFC) : Typologie des milieux étudies dans l'espace des paramètres biotiques. $G=$ groupe de milieux.

La seconde sous-unité (N1-2) comprend des espèces occupant la même position que la première, mais renferme des espèces qui peuvent être liées à des eaux courantes temporaires de montagne (torrent du Guedrouz) : Cosmarium anceps, $C$. tinctum, Netrium digitus, Closterium jenneri.

- Le noyau 4 est également subdivisé en deux sous-unités ; la première (N4-2) regroupe les espèces occupant une position périphérique éloignée de l'axe 1 et à l'opposé du noyau 1 , ce sont donc des taxons vivant dans des milieux permanents de plaine dont l'eau est caractérisée par un $\mathrm{pH}$ basique et une minéralisation relativement importante : Cosmarium variolatum, C. laeve var, messikommeri, Staurastrum paradoxum. La deuxième sous-unité (N4-1) regroupe des espèces eurytopes à faible signification structurale, qui occupent des positions proches de l'origine des axes 1 et 2 , ce sont donc des espèces considérées comme cosmopolites : Cosmarium laeve, Closterium acerosum, Staurastrum punctulatum. 
- Le noyau 2 est un ensemble diffus d'espèces dont la contribution aux deux premiers axes est faible. Elles se trouvent dans des biotopes de montagne, qu'ils soient courants (Oued Zat, Rhéraya, Ait Irène) ou stagnants (lac Oukaimeden, lac Tamda) et dont les eaux sont faiblement ou moyennement minéralisées, avec un pH légèrement basique (Groupe G2).

- Le noyau 3 individualise des espèces occupant une position périphérique positive de l'axe 2 à l'opposé du noyau précédent ; ces espèces sont caractéristiques des mares temporaires de Jbilet et de la Bahira (Groupe G3), peu profondes, turbides et fortoment chargées en matières en suspension (Cosmarium scopulorum, $C$. biretum et Closterium acerosum). L'axe 2 exprime, à nouveau, la turbidité des caux et leur charge en matières en suspension, ces deux paramètres étant liés à la profondeur des biotopes étudiès.

- Le noyau 5 est formé d'une seule espèce (Cosmarium pachydermum) caractéristique de la daya du douar Slalma dans les Jbilet orientales (TS8) (Groupe G5).

La typologie biologique (fig. 6) des écosystèmes ét udiés ressemble à celle obtenue à partir des paramètres abiotiques. Ceci montre que les populations algales de ces milieux reflètent de façon assez fidèle la composition chimique de l'eau.

\subsection{Evolution temporelle du peuplement desmidial}

Les données biotiques et abiotiques de 6 stations suivies régulièrement ont fait l'objet d'une analyse multivariée : deux dayas de montagne, Guedrouz (TS1) et Yagour (TS2) ; deux dayas de plaine, Jbilet (TS5) et Sedd El Messjoun (TS7) ; lac Oukaimeden (PS1) et bassin d'Agdal (PS4).

\subsubsection{DesmidieEs PLANCTONIQUES}

La classification hiérarchique des espèces a permis de distinguer 5 noyaux (fig. 7 ).

- Le noyau 1 (Pl) regroupe les espèces planctoniques des mares temporaires de montagne (Guedrouz et Yagour) caractérisées par des eaux faiblement minéralisées à $\mathrm{pH}$ légèrement acide. Ce noyau P1 peut être subdivisé en deux sous-unités ; la première, P1-1, correspond au groupe d'espèces récoltées au début de la mise en eau de la mare de Guedrouz avec Penium margaritaceum, Cosmarium abbreviatum, Cosmarium baffinensis et Slaurastrum crenulatum comme algues dominantes. La seconde sous-unité, P1-2, correspond au peuplement desmidial

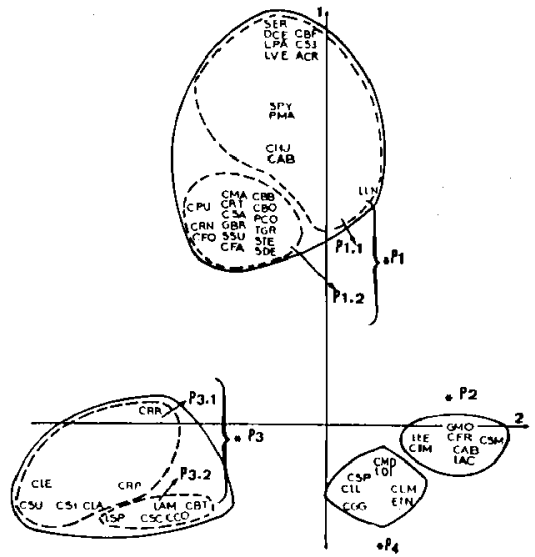

Fig. 7 : Analyse temporelle (AGC) des especes planctoniques : Projection des taxons dans le plan factoriel (1-2).

récolté lors de la phase précédant l'assèchement de la mare ; on observe la disparition de certaines espèces du début de la mise en eau (Penium margaritaceum) et l'apparition d'autres, telles Cosmarium subcapitulum et Pleurotaenium coronatum.

Le peuplement planctonique du début de mise en eau est plus riche en espèces que celui de la fin du cycle hydrologique de la mare.

- Le noyau 4 (P4) est opposé au premier sur l'axe 1 ; il regroupe les espéces planctoniques du bassin permanent de plaine, l'Agdal, caractérisé par des eaux alcalines et fortement minėralisées. Le peuplement desmidial est assez stable dans le temps. Il est dominé par Closterium dianae, Cosmarium granatum et Cosmarium laeve.

- Le noyau $2(\mathrm{P} 2)$ est situé à l'extrémité positive de l'axe 2 ; il regroupe les espèces planctoniques des échantillons du cycle annuel du lac de barrage de montagne : Oukaimeden. Le peuplement desmidial est également stable dans le temps. Il est dominé par : Gonatozygon monotaenium, Cosmarium formosulum et Cosmarium impressulum. Ces espèces caractérisent ici un milieu permanent profond et peu turbide. 
- Le noyau 3 (P3) se situe à l'opposé de P2 sur l'axe 2 ; il regroupe les espèces des mares temporaires des Jbilets (P3-1) et de la Bahira (P3-2) caractérisées par des eaux très peu profondes, turbides et très chargées en matières en suspension. Ce noyau est subdivisé en deux sous-unités ; la première, P3-1, regroupe les espèces récoltées lors de la mise en eau de la daya Boukricha ; Cosmarium latifrons est dominant, $C$. regnellii rare. La deuxième sous-unité, P3-2, correspond au peuplement planctonique de la mare Sedd El Messjoun, peuplement dominé par Closterium acerosum var. minus, Cosmarium scopulorum et $C$. biretum. Cette mare se caractérise par de fortes teneurs en chlorures.

Ces résultats montrent que, à l'exception des mares temporaires de montagne, le peuplement planctonique évolue peu au cours de la période d'étude.

\subsubsection{PERIPHYTON}

La classification hiérarchique individualise quatre groupements d'especes (fig. 8).

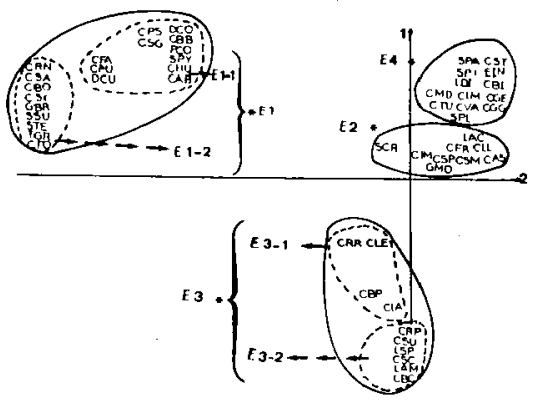

Fig. 8 : Analyse temporelle (AFC) des especes périphytiques : Projection des espèces dans le plan factoriel (1-2).

- Le noyau 1 (EI) est subdivisé en deux sousunités E1-1 et E1-2 ; il rassemble les espèces obtenues par expression des plantes aquatiques des mares de montagne faiblement minéralisées et légèrement acides. La sous-unité El-1 correspond aux espèces récoltées au départ de la mise en eau, lorsque la végétation macrophytique commence à se développer.
Ce peuplement est dominé par Cosmarium humile, C. abbreviatum, C. pusillum et C. botrytis. La deuxième sous-unité E1-2 renferme les desmidiées obtenues à partir de l'expression des plantes aquatiques (Marsilea, lsoetes) qui se développent pendant la phase précédant l'assèchement de la mare. On note l'apparition de Gonatozygon brebissonii, Teilingia granulata, Staurastrum tetracerum, Cosmarium boeckii et $C$. subcapitulum.

Le peuplement périphytique de la phase précédant l'assèchement total de la mare est plus riche en espèces que celui du début de la mise en eau.

- Le noyau 4 (E4) est opposé au noyau 1 sur l'axe 2 ; il renferme un peuplement desmidial presque stable dans le temps dans le bassin de l'Agdal. Il correspond aux especes qui trouvent au voisinage des plantes aquatiques de ce milieu un support idéal pour leur développement et leur persistance avec dominance du genre Cosmarium (Coesel 1982).

- Le noyau 2 (E2) correspond au peuplement périphytique du lac Oukaimeden; la majorité des espèces récoltées par l'expression des plantes aquatiques est asse $z$ proche de l'origine des deux axes factoriels 1 et 2 (Gonatozygon monotaenium, Cosmarium impressulum). Les résultats témoignent d'une certaine stabilité des populations de desmidiées périphytiques au cours de l'année d'étude, stabilité liée à la présence permanente d'une ceinture de végétation dans ce biotope.

- Le noyau 3 (E3) est situé à l'opposé du précédent sur l'axe 1 ; il décrit le peuplement desmidial des dayas des Jbilets (E3-1) et de la Bahira (E3-2). Il regroupe d'une part des espèces du début du cycle hydrologique lorsque les eaux sont limpides (Cosmarium regnellii et $C$. levinotabile) et d'autre part des espèces supportant des eaux très turbides ainsi que de très hautes valeurs en chlorures (Cosmarium scopulorum, $C$. biretum et Closterium acerosum).

\section{Conclusion}

Différentes approches dans l'analyse des résultats ont permis de mettre en évidence divers types de populations de desmidièes qui reflètent les caractéristiques physicochimiques des biotopes étudiés. Les desmidiées sont de bons bioindicateurs de la qualité des eaux. Par ailleurs, la flore desmidiale de la région de Marrakech présente différents aspects que l'on peut résumer de la façon suivante :

- Les espèces sont, pour la plupart, cosmopolites. 
- Le genre Micrasterias est absent. Les genres Cosmarium et Closterium prèsente une grande diversité spécifique, soit 73 et 37 espèces. Selon Coesel (1983), le genre Micrasterias est caractéristique des eaux oligotrophes.

- Certaines espèces s'adaptent à des eaux très minéralisées et surtout chlorurées (jusqu'à $400 \mathrm{mg} / \mathrm{l}$ ). Par exemple, dans le chott Sedd El Messjoun, on observe un peuplement desmidial dominé par Cosmarium scopulorum, C. biretum et Closterium acerosum. Cosmarium biretum est donné comme ubiquiste par d'Hollander (1980). La variété minus de Closterium acerosum est diversement considérée par les auteurs ; pour certains, comme Fjerdingstadt (1964), Couté \& Rousselin (1975), Cardinal (1979) et D'Hollander (1980), elle a une très large répartition géographique mais en eau calcaire ; pour Caljon (1983), cette variété est typique des eaux oligohalines ou polluées par la matière organique.

- Une grande richesse spécifique différencie les dayas de haute montagne (milieux peu minéralisés) des autres milieux étudiès.

- Dans les mares temporaires (dayas), la stratégie de survie des desmidiées s'appuie essentiellement sur la reproduction sexuée avec formation de zygotes pouvant résister à des périodes d'assèchement de 3 à 8 mois.

- 82 taxons, soit $61 \%$ de la flore desmidiale inventoriée, sont signalés au Maroc pour la première fois ; c'est donc un apport important du point de vue de la répartition géographique de ce groupe d'algues, mais cela montre aussi que de nouvelles études algologiques doivent être entreprises dans cette région.

\section{Remerciements}

Nous remercions Monsieur Alain Dauta pour sa contribution à l'accomplissement de ce travail.

\section{Travaux cités}

Belloc ( $F$.) . 1893. - Recherches sur les algues d'eau douce et des eaux thermales et des eaux salées d'Algérie, de Tunisie et du Maroc, suivie d'une liste des diatomées fossiles et d'un apercu de la flore diatomique marine littorale. Rev. Biol. $N$. de la France se A. : 1-15.

Belloc (F.). 1896, - Aperçu de la flore algologique d'Algérie et du Maroc et de quelques lacs de Syrie. A,F,A,S. Carthage : 406-412.

Benzecri (J.P.) \& Lebart (L.). 1970. - Distance distributionnelle et métique. P.U.B.L. Labo. Stat. Math. : I.S.U.P. - 3e éd. : $86 \mathrm{p}$.

Bourrelly (P.). 1972. - Les algues d'eau douce. Initiation à la systématique. Tome 1 : les algues vertes. Boubée et Cie éd., Paris : $572 \mathrm{p}$.

Bourrelly (P.)\& Gayral (P.). 1950. - Une nouvelle volvocale, Tingitanella pasqualinii, nov. gen. nov. sp. Bull. Sci. $N$. Maroc, $30: 117.120$.
Bourrelly (P.) \& Gayral (P.). 1951a. - Eugleniens Marocains rares ou nouveaux. Bull. Soc. Sci. Maroc: $3 ! \mathrm{p}$.

Bourrelly (P.) \& Gayral (P.). 195Ib. - Un gtoupement rhéophile dans un Oued du grand Atlas. $70^{c}$ Congrès A.F.A.S., $5: 1-3$.

Caljon (A.G.). 1983. - Brackish water phytoplancton of the Flemish lowland. Developments in Hydrobiol. $\mathrm{n}^{\circ} 18$, Dr. $\mathrm{W}$. Junk Publ., Dordrecht, Pays-Bas : 272 p.

Cardinal (C.). 1979. - Algues planctoniques du bassin de la Seine (à l'exception des Cyanophycées et des Diatomées). Bull. Mus. Hist. Nat. Paris, 4c sér, 1. Section 13, $4: 285-327$.

Coesel (P.F.M.). 1982. - Structural characteristics and adapLations of Desmid communities. J. Ecol. 70: 163-177.

Coesel (P.F.M.). 1983. - The signifiance of Desmids as indicators of the trophic status of freshwater. Schweiz. Z. Hydrol., 45, 2 : 388-393.

Cordier (B.). 1969. - L'analyse factorielle des correspondances. Thèse de $3 \mathrm{c}$ cycle, Rennes : $34 \mathrm{p}$.

Couté (A.) \& Rousselin (G.). 1975. - Contribution a l'ětude des algues d'eau douce du moyen Niger (Mali). Bull. Mus. Hist. Nat, , 3e sér., Bot., $21: 73-176$.

Darley (J.) \& Couté (A.). 1983. - Cosmarium marocanwm nov. sp. (Chlorophyta, Zygophyceae, Desmidiale, Desmidiacée). Cryptogamie-Algologie, 5, 1-2:99-103.

Derraz (M.). 1987. - Influence des facteurs environnementaux sur l'évolution de la qualité des eaux de la retenue de Oued El Makhazine (Maroc). Thèse de $3^{6}$ cycle, Toulouse : $95 \mathrm{p}$.

D'Hollander (E.). 1980. - Le phytoplancton du massif du Taganant( Mauritanie) à l'exclusion des diatomées. Bull. Roy. Bot. Belg., $113: 75-90$.

Fjerdingstad (E.). 1964. - Pollution of streams estimated by bentha! phytomicroorganisms. 1. Saprobic system based on communities of organisms and ecological factors. Internat. Rev. Ges. Hydrobiol., $49: 63-131$.

Gattefosse (J.) \& Werner (R.G.). 1935. - Catalogus algarum maroccanum adhucognitorum. Bull. Soc. Hist. Nat. Maroc, $15: 72-107$

Gauthier-Lièvre (L.). 1931. - Recherches sur la flore des eaux continentales de l'Afrique du Nord. Bull. Soc. Hist. Nat. Afr. Nord, Mem. hors série : $300 \mathrm{p}$.

Gauthier-Lievre (L.). 1951. - Notes sur quelques algues nordafricaines, Bull. Soc. Hist. Not. Afr. Nord, $42 \div 1-20$.

Gayral (P.). 1954. - Recherches phytolimnologiques au Maroc. Thèse Doct. ès Sci. Nat. Paris : 301 p.

Harriot (P.). 1913. - Algues d'eau douce du Maroc. Bull. Soc. Bot. $60: 40-43$.

Lund (J.W.G.). 1971. - The seasonal periodicity of three plancIonic Desmids in Windermere. Mitt. Intern. Ver. Limnol., $19: 3-25$.

Marqué (C.) \& Dauta (A.). 1987. - Production primaire d'une mare temporaire de la meseta occidentale marocaine. Siciences de l'eau, 6 (3) : 299-310.

Mercier (M.). 1922. - Note sur les algues vertes rencontrées dans la région de Casablanca. Maroc Médical, $8: 303-304$.

Mouhri (K.). 1987. - Répartition et association des Desmidiées (Chlorophycées, Zygnematales) dans différentes zones géographiques de la région de Marrakech (Maroc). Thèse de 3 e cycle, Univ. Cadi Ayyad, Fac. Sci, Marrakech : 152 p.

Sasson (A.). 1959. - Recherches écologiques et biologiques sur les algues d'une mare temporaire. Trav. Inst. Sci. Chérif. sér. Bot., $\mathbf{n}^{\circ}$ 17, Rabat : 103 p. 
Somers (D.). 1972. - Contribution à la flore des algues de Dayet Iffer et de l'Aguelmene Sidi Ali, deux lacs du Moyen Atlas au Maroc. Bull. Soc. Sci. Nat. Phys. Maroc, 52 : 31-46.

Talling (J.F.). \& Talling (I.B.). 1965. - The chemical composition of African lakes Waters. Int. Rev. Ges. Hydrobiol., 50 (3) : 421-463.
Utermohl (H.). 1958. - Zür Vervollkommung des quantitaven Phytoplankton Methodik. Mitt. Intern. Ver. Limnol., 9 : 1-38.

Van Oye (P.). 1949. - Nouvelles données sur les desmidiées des environs de Matadi. Hydrologia, 1 : 282-308.

Werner (R.G.). 1934. - Contribution à la flore cryptogamique du Maroc. Bull. Soc. Sci. Nat. Maroc, 14 : 151-155. 